\title{
Wolbachia strain wPip yields a pattern of cytoplasmic incompatibility enhancing a Wolbachia-based suppression strategy against the disease vector Aedes albopictus
}

Maurizio Calvitti ${ }^{*}$, Riccardo Moretti ${ }^{1}$, Amanda R Skidmore ${ }^{2}$ and Stephen L Dobson ${ }^{2}$

\begin{abstract}
Background: Cytoplasmic incompatibility $(\mathrm{Cl})$ is induced in nature by Wolbachia bacteria, resulting in conditional male sterility. Previous research demonstrated that the two Wolbachia strains (wAlbA and wAlbB) that naturally co-infect the disease vector mosquito Aedes albopictus (Asian tiger mosquito) can be replaced with the wPip Wolbachia strain from Culex pipiens. Since Wolbachia-based vector control strategies depend upon the strength and consistency of $\mathrm{Cl}$, a greater understanding is needed on the $\mathrm{Cl}$ relationships between wPip, wAlbA and wAlbB Wolbachia in Ae. albopictus.

Methods: This work consisted of a collaborative series of crosses carried out in Italy and in US to study the Cl relationships between the "wPip" infected Ae. albopictus strain (ARwP) and the superinfected SR strain. The Ae. albopictus strains used in Italian tests are the wPip infected ARWP strain (ARWP $P_{T T}$ ), the superinfected SR strain and the aposymbiotic AR strain. To understand the observed pattern of $\mathrm{Cl}$, crossing experiments carried out in USA focused on the study of the $\mathrm{Cl}$ relationships between ARwP (ARWPus) and artificially-generated single infected lines, in specific HTA and HTB, harbouring only wAlbA and wAlbB Wolbachia respectively.

Results: The paper reports an unusual pattern of $\mathrm{Cl}$ observed in crossing experiments between ARwP and SR lines. Specifically, ARwP males are able to induce full sterility in wild type females throughout most of their lifetime, while crosses between SR males and ARWP females become partially fertile with male aging. We demonstrated that the observed decrease in $\mathrm{Cl}$ penetrance with SR male age, is related to the previously described decrease in Wolbachia density, in particular of the wAlbA strain, occurring in aged superinfected males.

Conclusions: The results here reported support the use of the ARWP Ae. albopictus line as source of "ready-made sterile males", as an alternative to gamma radiation sterilized males, for autocidal suppression strategies against the Asian tiger mosquito. In addition, the age dependent $\mathrm{Cl}$ weakening observed in the crosses between SR males and ARwP females simplifies the downstream efforts to preserve the genetic variability within the laboratory ARwP colonies, to date based on the antibiotic treatment of wild captured superinfected mosquitoes, also reducing the costs.
\end{abstract}

Keywords: Cytoplasmic incompatibility, Wolbachia pipientis, Incompatible insect technique, Transinfection, Aedes albopictus

\footnotetext{
*Correspondence: maurizio.calvitti@enea.it

'Laboratory of Sustainable Management of the Agro-ecosystem, ENEA (Italian National Agency for New Technologies, Energy and Sustainable Economic Development), Via Anguillarese, 310, 00123, Rome, Italy

Full list of author information is available at the end of the article
} 


\section{Background}

Aedes (Stegomyia) albopictus (Diptera: Culicidae) (Asian tiger mosquito) is known as a mosquito species with an invasive behavior and a competent vector of various dangerous viruses [1-3]. In a few years since its arrival in Italy, Ae. albopictus has gained the position of the most important public health vector species and is at the top of the noxious species list $[4,5]$. The recent occurrence of autochthonous epidemics of Chikungunya and Dengue viruses in southern Europe [6,7] transmitted by Ae. albopictus, seems to confirm that the currently applied mosquito control methods (larval control, source reduction, and community participation) are not sufficient to keep the mosquito adult density below the epidemic risk threshold [8]. This was the main reason that stimulated the start of research for the development of a Sterile Insect Technique (SIT) program in Italy [9]. Several characteristics make Ae. albopictus a suitable candidate for SIT application, as follows: it is a recently introduced species with population showing a low genetic variability [10], it mainly colonizes urban areas while showing low aptitude to establish in rural and natural areas, it has a low active dispersal activity [11-13], and it is relatively easy to manage under mass rearing and artificial conditions [9]. Nowadays interest in SIT for vector control has resurfaced, driven also by the availability of new technologies that have the potential to provide significant improvements in costeffectiveness for SIT [14]. Although males of Ae. albopictus may be sterilised through ionizing radiations, by exposing mature pupae to a dose of 30-40 Gy $\gamma$-rays [15], the technique requires laborious handling procedures to prepare pupae for irradiation and transportation, in addition to the need for a radiation source, which is an expensive tool that needs an infrastructure requiring a substantial regulatory framework. Developing alternative technologies to produce "ready-made sterile males", avoiding sterilization with gamma rays, could improve the overall competitiveness of the released insects with a consequent improvement in program efficiency and a significant decrease in costs.

In the last two decades, scientists have given an increasing level of attention to Wolbachia pipientis Hertig (Alphaproteobacteria, Rickettsiales) [16], a widespread intracellular bacterium [17] able to manipulate host reproduction [18]. Cytoplasmic incompatibility (CI) is the most commonly detected type of Wolbachia-induced reproductive alteration in insects [19]. When a population contains individuals with different Wolbachia infection types (infected/uninfected or infected by different Wolbachia strains) their crosses can be (i) compatible and produce viable offspring; (ii) incompatible in both directions and produce infertile eggs (a phenomenon called bidirectional CI) or (iii) incompatible in one direction while the reciprocal cross is fertile (unidirectional
CI). While the genetic and biochemical mechanisms of $\mathrm{CI}$ are not known, the cytological effects are clear [20]. Sperm that are "modified" by Wolbachia in the testes show abnormal processing following fertilization of the egg, if the appropriate Wolbachia strain is not present in the egg to "rescue" the modification [21].

These attributes are now being studied by many research groups with the aim of developing new technologies and strategies to achieve significant improvements in pest and vector control. Wolbachia-mediated CI has been proposed as a strategy for insect control via two approaches: (1) using CI to cause sterility for a mass male release strategy analogous to sterile insect technique and consequently named "Incompatible Insect Technique" (IIT) [14,22-25], or (2) using the reproductive advantage afforded by Wolbachia-induced CI as a tool for a population replacement strategy, driving desired phenotypes (e.g., lower affinity for pathogens) into medically important mosquito populations [26-29]. Both of these approaches require a method to artificially transfer Wolbachia, generating new patterns of CI [30]. In 2010, a transinfected line $(\mathrm{AR} w \mathrm{P})$ of Ae. albopictus was generated by removing the naturally occurring co-infection of $w$ AlbA plus $w$ AlbB and microinjecting in the aposymbiotic eggs the wPip Wolbachia strain from Culex pipiens molestus (Diptera: Culicidae) [31]. The new symbiosis was shown to be stable and efficiently transmitted from females to their offspring. Since Wolbachia-based vector control strategies rely on the strength and consistency of $\mathrm{CI}$, a greater understanding is needed of the CI pattern resulting between the $\mathrm{AR} w \mathrm{P}$ line and the naturally occurring infection types in Ae. albopictus. The main goal of this work was to characterize and explain the pattern of CI displayed in crosses between the $\mathrm{AR} w \mathrm{P}$ and $\mathrm{SR}$ mosquito lines and evaluate its implications in the development of Wolbachia-based strategy against the Asian tiger mosquito.

This was done by analyzing the egg hatching in crossing experiments involving five laboratory mosquito lines: i) the transinfected $\mathrm{AR} w \mathrm{P}$ (harbouring $w$ Pip); ii) the naturally superinfected SR strain (harbouring both the $w$ AlbA and $w$ AlbB infection types); iii) the artificially single-infected HTA strain (infected with wAlbA only); iv) the artificially single-infected HTB strain (infected with $w$ AlbB only); and v) the AR strain that has had its Wolbachia infection removed (i.e. aposymbiotic). In addition to examining young males, for some strains we evaluated also the effects of male aging on $\mathrm{CI}$, through crosses involving old males.

\section{Methods}

\section{Mosquito strains and rearing conditions}

This work consisted of a collaborative series of crosses: one series (Series I) conducted at the Laboratory of Sustainable Management of the Agro-ecosystems of ENEA 
(Rome, Italy); the other series (Series II) at the Department of Entomology, University of Kentucky (Lexington, Kentucky, USA). The Ae albopictus strains used in Italian tests are the $w$ Pip infected $\mathrm{AR} w \mathrm{P}$ strain $\left(\mathrm{AR} w \mathrm{P}_{\mathrm{IT}}\right)$, the superinfected SR strain and the aposymbiotic AR strain, as defined in a previous work [31]. In order to avoid genetic depression, the three lines have been periodically outcrossed with wild superinfected males, following antibiotic treatment for Wolbachia removal [32,33]. The crossing experiments carried out in USA focused on the study of the CI relationships between $\mathrm{AR} w \mathrm{P}\left(\mathrm{AR} w \mathrm{P}_{\mathrm{US}}\right)$ and artificially-generated single infected lines, in specific HTA and HTB, harbouring only wAlbA and wAlbB Wolbachia respectively. $\mathrm{AR} w \mathrm{P}_{\text {US }}$ colony originated from a stock of about 5,000 eggs produced by the $\mathrm{AR} w \mathrm{P}_{\mathrm{IT}}$ strain and shipped to the University of Kentucky in 2010.

All colonies, both in Italy and USA, were maintained as previously described [32]. Since temperature of water used for larval rearing may influence Wolbachia density and CI penetrance [34], care was used to keep the water temperature between 25 and $27^{\circ} \mathrm{C}$.

When testing for $\mathrm{CI}$, potential confounding effects influencing fertility of the crossing experiments, such as the nuclear background of the host, have to be limited by the experimental design [35]. Since ARwP $\mathrm{P}_{\text {US }}$ and HTA-HTB had been generated from wild type mosquito strains having a different geographic origin, HTA and HTB lines (originated from US "Hou" strain) and ARw $\mathrm{P}_{\mathrm{US}}$ were outcrossed for 5 consecutive generations with aposymbiotic AR males obtained from a stock of eggs also shipped to US from Italy.

The HTA line was generated using a previously described microinjection procedure [36]. In brief, aposymbiotic embryos (HT1 strain) [32] were microinjected with cytoplasm containing Wolbachia from wild type Ae. albopictus embryos (Hou strain, Texas 1986). Adult females developing from microinjected eggs were mated with aposymbiotic males (HT1 strain), blood fed, isolated and allowed to oviposit individually. Iso-female lines were generated from hatching egg broods that originated from females in which Wolbachia was detected. This procedure was repeated until maternal transmission rates of Wolbachia reached $100 \%$ for more than three generations. For the HTA strain, selection was repeated for eight generations. After the second generation, all iso-females tested were PCR positive for Wolbachia using the Wolbachia molecular diagnosis protocol described below. Additional tests with clade-specific primers demonstrated that infected females were positive for A-clade specific Wolbachia (328 F, 691R primer set). All tests using Bclade specific primers (183 F, 691R primer set) were negative, indicating the loss of one of the Wolbachia types. Following the eighth generation, iso-female selection was stopped and the line was maintained using a generation specific (non-overlapping) rearing scheme in which no selection was used. Periodic (every three generations) A-clade specific Wolbachia primers checks were performed to ensure the stability of Wolbachia infection levels. HTB line was obtained following a similar procedure, as described in a previous work [37].

\section{Wolbachia molecular diagnosis}

PCR assays were performed to check that all males and females mosquitoes used in the experiments had the expected infection type. Molecular discrimination of uninfected from infected males was performed by the diagnostic wsp primers (81 F-691R) that amplify a region of the gene encoding the Wolbachia outer surface protein (wsp) and allow for a broad identification of Wolbachia strains [38]. Wolbachia strains $w$ Pip and wAlbB can be easily identified by the same specific set of primers (183 F, 691R) [39] when they live separated in their natural hosts $(C x$. pipiens and Ae. albopictus respectively). Here we were faced with the need to discriminate $w$ Pip from $w$ AlbB infected individuals to ascertain the absence of contaminations. For this purpose we designed the following specific set of primers: $w$ PF $\left(5^{\prime}-\right.$ CGACGTTAGTGGTGCAA CATTTA $-3^{\prime}$ ) and $w$ PR (5' AATAACGAGCACCAGCAA AGAGT-3') by which we were able to specifically amplify the $w s p$ region of the $w$ Pip Wolbachia strain.

DNA was extracted from individual mosquitoes by dissecting and homogenizing ovaries or testis of adults in $100 \mu \mathrm{l} \mathrm{STE}$ with $0.4 \mathrm{mg} / \mathrm{ml}$ proteinase $\mathrm{K}$ [4]. The PCR cycling procedure used was: $94^{\circ} \mathrm{C}$ for $5 \mathrm{~min}$ followed by 35 cycles of $94^{\circ} \mathrm{C}$ for $30 \mathrm{~s}, 55^{\circ} \mathrm{C}\left(54^{\circ} \mathrm{C}\right.$ for the $w \mathrm{P}$ primers $)$ for $30 \mathrm{~s}, 72^{\circ} \mathrm{C}$ for $40 \mathrm{~s}$ and a single final step at $72^{\circ} \mathrm{C}$ for $10 \mathrm{~min}$. Amplified fragments were electrophoresed on $2 \%$ agarose gels, stained with ethidium bromide $(1 \mu \mathrm{g} / \mathrm{ml})$ and visualized under ultraviolet light. DNA template quality was assessed by amplifying a fragment of the insect mitochondrial cytochrome oxidase I (COI) DNA, using the primers CI-J-1751 and CI-N- 2191 [40].

\section{Crossing experiments}

The following series of 7 crosses (female $x$ male) were set up in Italy: 1) $\mathrm{SR} x \mathrm{SR}, 2) \mathrm{AR} w \mathrm{P}_{\mathrm{IT}} \mathrm{x} \mathrm{AR} w \mathrm{P}_{\mathrm{IT}}$, 3) $\mathrm{AR} \mathrm{x}$ $\mathrm{AR}$, 4) $\left.\mathrm{AR} \times \mathrm{SR}, 5) \mathrm{AR} w \mathrm{P}_{\mathrm{IT}} \mathrm{x} \mathrm{SR}, 6\right) \mathrm{AR} \times \mathrm{AR} w \mathrm{P}_{\mathrm{IT}}$, 7) $\mathrm{SR} \times \mathrm{AR} w \mathrm{P}_{\mathrm{IT}}$.

$\mathrm{CI}$ relationships between $\mathrm{AR} w \mathrm{P}, \mathrm{SR}$ and $\mathrm{AR}$ mosquito strains had been previously observed and shown to result in a pattern of bidirectional incompatibility for crosses between $\mathrm{AR} w \mathrm{P}$ and SR mosquito lines and unidirectional CI by crossing $\mathrm{AR} w \mathrm{P}$ with $\mathrm{AR}$ and $\mathrm{SR}$ with $\mathrm{AR}$ [31-33]. In this work, we examined the variable "male age" for crosses of SR and AR $w$ P males with virgin 2-4 d old SR, AR $w$ P and AR females. For the objectives of this work, female age was kept constant in all crosses (2-4 d). 
For Series I experiments male age was assessed from the emergence (= Day 0). Groups of males were aged 3, 11,19 and 27 days $( \pm 1)$. For each cross type, 20 females and 20 males were kept together in mating cages $(40 \times$ $40 \times 40 \mathrm{~cm}$ ) over a $24 \mathrm{~h}$ period. Subsequently, female groups were allowed to feed on anesthetized mice, in accordance with the Bioethics Committee for Animal Experimentation in Biomedical Research and following procedures approved by the ENEA Bioethical Committee. Gravid females were then removed from the mating cages and transferred to a new cage (oviposition cage) and provided with oviposition devices as previously described [31]. Eggs were counted and stored for $5 \mathrm{~d}$ before allowing them to hatch, by the immersion in a nutrient broth stimulating hatching [9]. In crosses with no egg hatch, females were dissected to check for the presence of spermatozoa. Males were kept in the original cages. After removing the first groups of females, a new cohort of virgin females was added to the cages containing males at 1:1 female:male ratio. Following this procedure, the CI relationship between the different Wolbachia infections was investigated under conditions similar to those common to a release of young incompatible males, getting older, more experienced and consuming their sperms in the field. This cycle was repeated for the four male age-classes.

The general protocol described above was used also for Series II experiments set up to study the CI relationships between the ARwP line and the two single-infected strains HTA and HTB. The following 7 crosses (female $x$ male) were set up in US: 1) $\mathrm{AR} w \mathrm{P}_{\mathrm{US}} \mathrm{x}$ HTA, 2) HTA x AR $w \mathrm{P}_{\mathrm{US}}$, 3) $\mathrm{AR} w \mathrm{P}_{\text {US }} \times \mathrm{HTB}$, 4) HTB $x$ AR $w \mathrm{P}_{U S}$, 5) $\mathrm{AR} w \mathrm{P}_{U S} \times \mathrm{AR} w$ $\left.\mathrm{P}_{\mathrm{US}}, 6\right)$ HTA $x$ HTA, 7) HTB $x$ HTB. The design of the latter crosses did not include male age and all crosses consisted of young (2-4 d old) males. Four cage replications were set up for each crossing type.

At the end of the crossing experiments, the infection status of the males was checked (by PCR assays) to verify that all had the expected infection type (to avoid an incorrect interpretation of the results possibly due to the rare presence of aposymbiotic individuals among males of an infected line). Molecular analysis was also performed on any males found dead in the cages to determine their symbiotic status.

\section{$\mathrm{Cl}$ computation and statistics}

Calculation of CI expression was based upon the mean egg hatch rate found in incompatible crosses in comparison with the results from the compatible crosses (e.g. $\mathrm{AR} w \mathrm{P} \times \mathrm{AR} w \mathrm{P}, \mathrm{SR} \times \mathrm{SR}, \mathrm{HTB} \times \mathrm{HTB}, \mathrm{HTA} \times \mathrm{HTA})$, using the $\mathrm{CI}_{\text {corr }}$ index [41]. This index allows the exclusion of embryonic mortality observed in compatible crosses and male age effects that are not due to CI expression.

Within the different crossing types egg-hatching data and CI values were compared in relation to male ages.
Normality of egg-hatching data was examined by D'Agostino and Pearson omnibus normality test using Prism 5 (Graphpad software). Significant differences among mean egg hatch rates were tests by analysis of variance (ANOVA) on arcsin sqrt transformed data. A statistical comparison was then performed by Newman-Keuls Multiple Comparison Test $(\alpha=0.05)$. Paired $t$ test was also used to analyze differences in mean egg hatching rates between two different groups.

\section{Results}

\section{Male age effects on hatch rate and $\mathrm{Cl}$ expression}

In all crosses between males and females harbouring the same Wolbachia infection type, the mean hatch rate was initially high $(80.0 \pm 6.0 \%$ to $86.2 \pm 4.7 \%$ hatch) (Table 1$)$. However, starting from the 3rd male age class, we observed a significant decline in egg hatch within each of the compatible crosses. In the $19 \pm 1 \mathrm{~d}$ male age class, egg hatch had significantly fallen to $46.4 \pm 10.30 \%$ in the AR $w \mathrm{P}_{\text {IT }}$ (ANOVA: $F=16.65$; d.f. $=12 ; P<0.001$ ), to $47.7 \pm 7.5 \%$ in AR (ANOVA: $F=10.69$; d.f. $=12 ; P=0.001$ ) and to $63.2 \pm 10.2 \%$ in SR mosquito line (ANOVA: $F=23.01$; d.f. $=12 ; P<0.001)$. A further decrease was observed with the males of the fourth age class. This general trend agrees with a gradual decrease of the insemination capacity as males get older already reported in previous works [33-42].

As shown in Table 1, all the crosses involving males of the first age class and females with a different infection status were characterized by a complete egg hatch failure. This result did not significantly change with male aging in three out of four incompatible crosses. Only in the cross between $\mathrm{AR} w \mathrm{P}_{\mathrm{IT}}$ females and $\mathrm{SR}$ males the percentage of eggs hatching increased significantly starting from the second age class (ANOVA: $F=150.00$; d.f. $=12 ; P<0.001$ ) and reaching values close to $20 \%$ with the oldest males. Consequently in this cross type $\mathrm{CI}_{\text {corr }}$ decreased to about $50 \%$ of that observed with 3 days old males (100\%) (Figure 1).

\section{$\mathrm{Cl}$ in crosses between single-infected lines}

The mean egg hatch values observed in the $\mathrm{AR} w \mathrm{P}_{\mathrm{US}} \mathrm{com}-$ patible crosses of Series II were lower than that observed in the Series I crosses $(45.0 \pm 22.2$ and $84.3 \pm 7.9 \%$ respectively at the first age class) (Table 2). A low hatch rate $(44.7 \pm 13.7 \%)$ was also observed in compatible crosses of the HTA line while HTB was the single infected line showing the highest egg fertility $(66.0 \pm 12.4 \%)$.

As shown in Table 2, crosses between the $\mathrm{AR} w \mathrm{P}_{\mathrm{US}}$ strain and the single infected lines showed low egg hatch levels and high $\mathrm{CI}_{\text {corr }}$ values (close to $100 \%$ ) with one exception, in the cross between $\mathrm{AR} w \mathrm{P}_{\mathrm{US}}$ females and males with the wAlbB infection only (i.e. HTB line) $\mathrm{CI}_{\text {corr }}$ weakened to $55.8 \%$ (Student's test, $\alpha=0.05 ; t=2.03$ ), corresponding to a $19.9 \pm 5.5$ percentage of eggs hatching. Reciprocal crosses 
Table 1 Percent egg hatch from crosses between Ae. albopictus lines, in dependence of male aging

\begin{tabular}{|c|c|c|c|c|}
\hline \multirow[t]{2}{*}{ Cross type $(q \mathbf{X} \precsim)$} & \multicolumn{4}{|c|}{ Percent egg hatch (mean \pm SD) at different male ages (days \pm 1 ) } \\
\hline & $3 \mathrm{~d}$ & $11 \mathrm{~d}$ & $19 \mathrm{~d}$ & $27 \mathrm{~d}$ \\
\hline$S R \times S R$ & $86.2 \pm 4.7(2758)$ & $79.1 \pm 7.9(3001)$ & $63.2 \pm 10.0^{\mathrm{a}}(2476)$ & $45.8 \pm 9.9^{b}(2238)$ \\
\hline$A R w P_{I T} \times A R w P_{I T}$ & $84.3 \pm 7.9(2646)$ & $69.9 \pm 8.0(2960)$ & $46.4 \pm 10.0^{\mathrm{a}}(2253)$ & $38.0 \pm 15.8^{\mathrm{a}}(1944)$ \\
\hline$A R \times A R$ & $80.0 \pm 6.0(2344)$ & $70.0 \pm 12.3(1999)$ & $47.7 \pm 7.5^{\mathrm{a}}(2843)$ & $43.0 \pm 15.4^{\mathrm{a}}(1822)$ \\
\hline$A R \times S R$ & $0.0(2730)$ & $0.0(2738)$ & $0.5 \pm 0.7(3034)$ & $1.0 \pm 2.0(2258)$ \\
\hline $\mathrm{ARWP}_{\mathrm{IT}} \times \mathrm{SR}$ & $0.0(3142)$ & $4.0 \pm 0.8^{\mathrm{a}}(2754)$ & $18.0 \pm 2.4^{\mathrm{b}}(2456)$ & $19.2 \pm 8.1^{b}(2678)$ \\
\hline$A R \times A R W P_{\Pi}$ & $0.0(2669)$ & $0.3 \pm 0.5(3453)$ & $0.2 \pm 0.2(2113)$ & $0.0(2014)$ \\
\hline$S R \times A R w P_{I T}$ & $0.0(3681)$ & $0.0(2905)$ & $1.3 \pm 1.9(2882)$ & $0.0(2251)$ \\
\hline
\end{tabular}

Presented data show the results of the Series I experiments, carried out in Italy.

$\mathrm{SR}=w \mathrm{AlbA}+w \mathrm{AlbB}$ superinfected; $\mathrm{AR} w \mathrm{P}_{\mathrm{IT}}=w$ Pip infected; $\mathrm{AR}=$ aposymbiotic.

Number of total scored eggs are in parenthesis. Within a row, letters following the data indicate significant differences $(P<0.05)($ Anova-Newman-Keuls Multiple Comparison Test).

between individuals with the $w$ Pip ( $\left.\mathrm{AR} w \mathrm{P}_{\mathrm{US}}\right)$ and $w \mathrm{AlbA}$ (HTA) infections were bidirectionally incompatible. In contrast, reciprocal crosses between individuals with the $w$ Pip $\left(\mathrm{AR} w \mathrm{P}_{\mathrm{US}}\right)$ and $w \mathrm{AlbB}(\mathrm{HTB})$ infections showed full incompatibility in one direction only.

\section{Discussion}

Data obtained by studying the egg hatch rate and thus computing the level of $\mathrm{CI}$ in crosses between $\mathrm{AR} w \mathrm{P}_{\mathrm{IT}}$ and SR lines have provided interesting insights that suggest an unusual pattern of bidirectional $\mathrm{CI}$, changing partially to unidirectional as superinfected SR males get older. We observed that males harbouring the $w$ Pip Wolbachia strain remain strong $\mathrm{CI}$ inducers, despite their age and regardless of whether they mate with naturally superinfected or uninfected females. In contrast, in crosses of naturallysuperinfected males ( $\mathrm{SR}$ line) with $\mathrm{AR} w \mathrm{P}_{\mathrm{IT}}$ females, CI drops so that egg hatching increases to approximately $20 \%$ as males reach the fourth age class (Table 1).

A number of studies have documented that the strength of Wolbachia-mediated CI can decrease as males get older. For example, in Drosophila melanogaster Meigen (Diptera: Drosophilidae) this occurs with values ranging from $70-100 \%$ of CI expression, when the males are very young (1-2 days), to extremely low levels (4-5\% of CI) after males age 15 days [43]. The underlying mechanistic hypothesis is that Wolbachia density decreases with male aging $[44,45]$. In $C x$. pipiens, CI strength was not found to decrease with male age [46], while an increasing bacterial density was observed in the testes of older males [47], in disagreement with the model according to which the CI penetrance tends to decrease in old males directly proportional to the density of Wolbachia in the testes or sperm cysts in general [48].

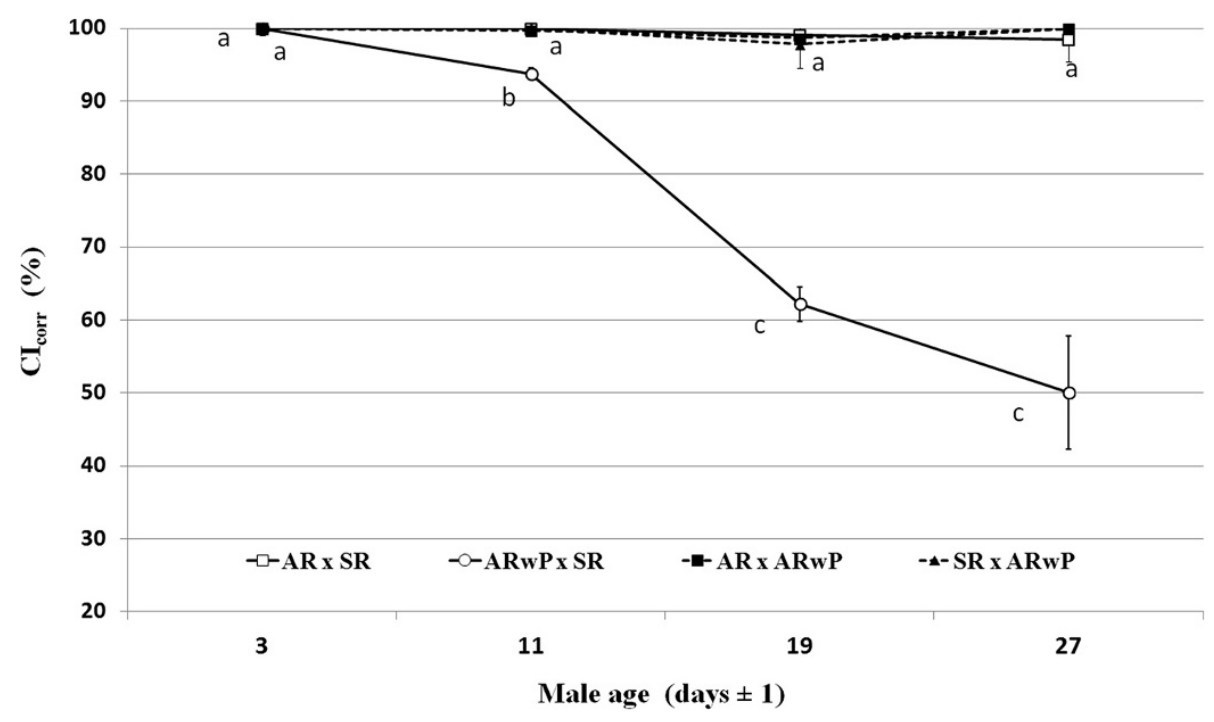

Figure 1 Estimated $\mathrm{Cl}$ levels in function of male ageing, $\mathrm{Cl}_{\text {corr }}$ (\%) [41] was estimated from egg hatching data observed in incompatible and compatible crossing types (female $\mathrm{x}$ male) involving SR (superinfected), AR (aposymbiotic) and ARwP (transinfected) Ae. albopictus lines. Different letters at each male age interval indicate significant differences $(P<0.05)$ (Anova-Newman-Keuls Multiple Comparison Test). 
Table 2 Percent egg hatch and $\mathrm{Cl}_{\text {corr }}$ level from crosses between Ae. albopictus lines different by their Wolbachia infection status

\begin{tabular}{|c|c|c|}
\hline Cross type $(+\mathbf{X} \precsim)$ & $\frac{\text { Percent egg hatch }}{\text { (mean } \pm \text { SD) }}$ & $\frac{\mathrm{Cl}_{\text {corr level }}}{\text { (mean } \pm \mathrm{SDCP}}$ \\
\hline$A_{R w P} \times A_{\text {S }}$ ARPus & $45.0^{\mathrm{a}} \pm 22.2(3210)$ & 0 \\
\hline HTA $\times$ HTA & $44.7^{\mathrm{a}} \pm 13.7(2976)$ & 0 \\
\hline $\mathrm{HTB} \times \mathrm{HTB}$ & $66.0^{b} \pm 12.4(2165)$ & 0 \\
\hline ARwPus $\times$ HTA & $0.9^{c} \pm 0.6(3266)$ & $98.0^{\mathrm{a}} \pm 1.5$ \\
\hline HTA $\times$ ARwPus & $0.0^{c}(3165)$ & $100^{\mathrm{a}}$ \\
\hline ARwP US $\times \mathrm{HTB}$ & $19.9^{d} \pm 5.5(2076)$ & $55.8 \pm 8.4^{b}$ \\
\hline HTB $\times$ ARWPus & $0.0^{c}(3241)$ & $100^{\mathrm{a}}$ \\
\hline
\end{tabular}

Presented data show the results of the Series II experiments, carried out in USA, using $3 \pm 1$ days old males. $\mathrm{AR} w \mathrm{P}_{\mathrm{US}}=w$ Pip infected; $\mathrm{HTA}=$ wAlbA single infected, $\mathrm{HTB}=$ wAlbB single infected. Number of total scored eggs are in parenthesis.

Within a column, letters following the data indicate significant differences $(\mathrm{P}<0.05)$ (Anova-Newman-Keuls Multiple Comparison Test).

With regard to Ae. albopictus, the data obtained in the present work confirm that the naturally superinfected males express a strong level of unidirectional CI towards the aposymbiotic AR line which does not undergo decreases with male aging, at least up to 26-28 days. In contrast, the same males show age dependent weakening of induced CI when crossed with $\mathrm{AR} w \mathrm{P}_{\mathrm{IT}}$ females (Figure 1). Data on CI relationships between $\mathrm{SR}$ and $\mathrm{AR}$ lines are consistent with previous reports [49] in which a strong CI expression was observed in all crosses between wild superinfected males and laboratory-reared uninfected or wAlbA (KOH line) infected young females. In addition, the same authors also reported a pronounced weakening of $\mathrm{CI}$ in old $\mathrm{KOH}$ males mated with aposymbiotic females. The $\mathrm{KOH}$ line is a natural single-infected line harbouring the wAlbA Wolbachia strain, similar to the artificial HTA described here. While the wAlbB density in Ae. albopictus remains constant over the mosquito life-time, the density of the wAlbA infection decreases gradually as the males get older [50,51].

In this work, we associated the decrease of CI observed in crosses between $\mathrm{AR} w \mathrm{P}_{\mathrm{IT}}$ females and $\mathrm{SR}$ aged males to the above described $w$ AlbA strain-dependent changes in Wolbachia density. However, to support this hypothesis, we needed to ascertain that males single-infected by the $w$ AlbB Wolbachia were only partially incompatible when crossed with $w$ Pip infected females. The change in Wolbachia density may explain the observed $\mathrm{CI}$ pattern if $(\mathrm{A}) \mathrm{B} \approx \mathrm{B}$ and if the $w$ AlbB infection alone causes partial incompatibility.

As an initial test to validate this model, we conducted crosses of single infected Ae. albopictus, artificially generated through microinjection, such that individuals carried either the $w \mathrm{AlbA}$ or $w \mathrm{AlbB}$ infection, with the $\mathrm{AR} w \mathrm{P}_{\mathrm{US}}$ line (Series II crosses). Consistently with model prediction, the $w$ AlbA infected males were observed to cause strong $\mathrm{CI}$ in crosses with $w$ Pip infected females, while the $w \mathrm{AlbB}$ infection caused partial CI in crosses with $w$ Pip infected females. In the reciprocal crosses, males infected with $w$ Pip induced strong incompatibility when mated with females that were single infected with either the $w$ AlbA or $w$ AlbB Wolbachia strains. According to the hypothesized model, mean $\mathrm{CI}_{\text {corr }}$ values found when crossing $\mathrm{AR} w \mathrm{P}_{\mathrm{IT}}$ females $\mathrm{x}$ old SR males (Figure 1) and $\mathrm{ARwP}_{\mathrm{US}}$ females $\mathrm{x}$ young HTB males (Table 2) were quite similar $(49.5 \pm 14.2 \%$ vs $55.8 \pm 12.8 \%)$.

The differences between Series I and II in egg hatch of the $\mathrm{AR} w \mathrm{P}$ line compatible crosses may be explained by a bottleneck resulting from the shipment between laboratories, by the different size of the colonies and by small variations in environmental factors, application of the rearing procedures and handlers. This discrepancy may also reflect an improvement in the ENEA strain quality (mainly occurred in the last two years) resulting from selection, outcrossing practices and enhancement of the rearing methods that will be the topic of a further article. In fact, egg hatching rates of $\mathrm{AR} w \mathrm{P}_{\mathrm{US}}$ compatible crosses resemble that reported in a previous work [31] for the $\mathrm{AR} w \mathrm{P}_{\mathrm{IT}}$ strain. However, these differences, even if significant, can not invalidate the scientific findings of the whole experimental plan, since the measurement of the $\mathrm{CI}_{\text {corr }}$ allowed us to take into account the misleading effects of the background mortality while interpreting the results coming from the two Series of experiments. Thus, we thought it was not necessary to wait for the establishment in the USA of AR $w$ P colonies showing levels of fertility similar to the Italian colony, since this process could take more than 1 year. Based on our preliminary results, the findings of this work will allow us to set up new high fitness ARwP colonies, within 2-3 generations of establishment (Moretti and Calvitti, unpublished data). A lower hatch rate was observed in compatible crosses of the HTA line if compared to egg hatching data reported for other singleinfected ( $w$ AlbA) strains established in other laboratories (i.e. $\mathrm{KOH}$ in the Islands of Koh Samui and Mauritius) [32-50]. Since HTA has been recently established in the laboratory, hypotheses to explain this observation include inbreeding effects associated with the establishment of isofemale lines (i.e. increased homozygosis of deleterious loci) and high mortality associated with the artificially generated single $w$ AlbB infection type. Introgression with males of uninfected mosquito lines could attenuate potential inbreeding effects as successfully demonstrated with HTB [34].

According to the mod-resc model [20], wPip is able to partially rescue the $w \mathrm{AlbB}$ mod function, while it is not able to rescue the $w$ AlbA mod. Differently, $w$ AlbB is a weak $\mathrm{CI}$ inducer towards $w$ Pip and cannot rescue the $w$ Pip mod function (asymmetrical CI). Such asymmetrical CI relationships had been previously reported in the $C x$. pipiens-Wolbachia system [52,53] as well as for $w \mathrm{Mel}$ and wRi Wolbachia strains [41]. The Wolbachia strain wAlbA 


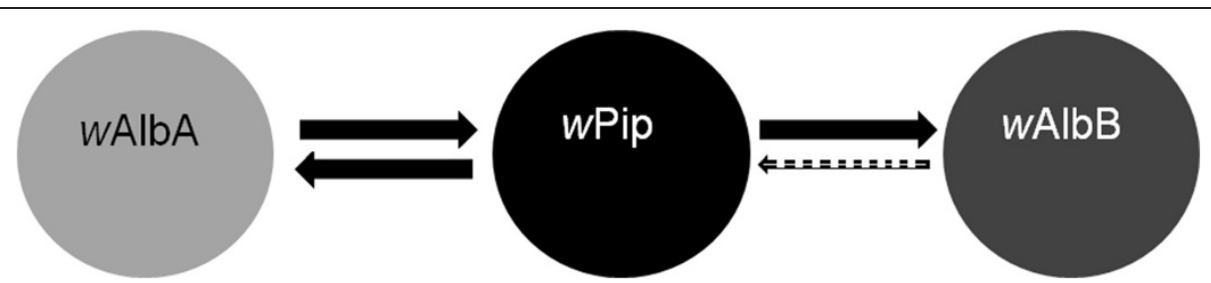

Figure $2 \mathrm{CI}$ relationships among wPip and Ae. albopictus native Wolbachia strains. The Wolbachia strain wPip is a strong Cl inducer towards WAlbA and WAlbB (both unable to rescue its mod function) While wPip it is not able to rescue the wAlbA mod function, it can partially rescue $w A$ IbB (arrows indicate $\mathrm{Cl}$ intensity).

is a strong CI inducer towards $w$ Pip and cannot rescue the $w$ Pip mod function (Figure 2).

\section{Conclusions}

The reported $\mathrm{CI}$ features are consistent with the traits desired for promising use of the AR $w$ P line as a suppression tool against Ae. albopictus. First, a persistent full CI in older males enforces the efficacy of any CI-based mosquito control strategy. This becomes more relevant given the report that Ae. albopictus males in La Réunion show an unexpectedly high mean life expectancy, ranging from 16.2 to 24.5 days [54]. Currently, the optimal radiation doses for a SIT programs against Ae. albopictus is chosen in such a way (30 rather than 40 Gy) that it balances induced sterility with the preservation of male competitiveness [15-55]. However, the more the irradiation dose is lowered, the more it is reasonable to assume that a potential recover of fertility could occur when males are still potentially competitive [56]. No recovery of fertility has been observed in $\mathrm{AR} w \mathrm{P}$ males tested up to 26 days.

Secondly, we know that the colonies of insects reared in the laboratory for subsequent field applications need to be periodically outbred to offset the effects of genetic adaptation to captivity and inbreeding depression [57-59]. For colonies of mosquitoes whose males are destined to be irradiated this problem is less relevant because there is no reproductive barrier between the insects of the colony and the wild types. Also for Wolbachia transinfected lines displaying a unidirectional CI pattern with uninfected wild populations [26-60] this problem does not arise because the males in nature are uninfected and therefore can be used to fertilize transinfected females.

In the case of $\mathrm{AR} w \mathrm{P}$ or other transinfected mosquito lines displaying bidirectional CI towards wild populations (wild males are incompatible with females of the colony), the outbreeding procedures require the treatment of wild males with antibiotics for Wolbachia infection removal [32] and "compatibility" restore. The use of antibiotics, very useful at laboratory scale, may be rather laborious, time consuming and not cost-effective to produce large amounts of "antibiotic cured males" under mass rearing conditions. The discovery that $\mathrm{AR} w \mathrm{P}$ females are partially fertile when mating with old wild Ae. albopictus males may simplify significantly the downstream efforts to preserve the genetic variability within the laboratory AR $w$ P colonies; in fact, it would be sufficient to release periodically in the colony ARwP females mated with wild males aged at least 15 days.

In one hand the interest for the $\mathrm{AR} w \mathrm{P}$ line in the IIT strategy against the Asian tiger mosquito has been increasing, in the other, new artificially generated infection types like Ae. albopictus harboring "wMel” [29], showing anti-viral (Chikungunya and Dengue) properties associated to limited fitness costs, are promoting the application of "population replacement" strategies. Recent trends in the application of a population replacement program suggest that a phase of population suppression should be performed to support a following male-biased release of the avirulent invading mosquito strain (i.e. $w$ Mel infected) minimizing any transient increase in disease risk or biting nuisance [61]. ARwP males, strong and persistent $\mathrm{CI}$ effectors, could be considered in the preliminary suppression phase of a population replacement program or for the application of suppression strategies in areas where there are not risks of pathogen transmission and consequently no need to replace a mosquito population. Although the $\mathrm{AR} w \mathrm{P}$ mosquito line appears to be relatively robust and suitable for mass rearing, research is in progress to achieve a further attenuation of the negative effects of the new Wolbachia infection on female reproductive parameters (fecundity and fertility), as well as mating competitiveness of males, which is being evaluated not only in the laboratory (Moretti \& Calvitti, unpublished data) but also in seminatural (confined greenhouses) and field conditions.

Competing interests

The authors declare that they have no competing interests.

\section{Author's contribution}

MC planned the work and performed Series I crossing experiments, analyzed data and wrote the first draft. RM contributed to the study design, performed crossing experiments and molecular analysis. ARS performed Series II of the crossing experiments and generated the HTA mosquito line. SLD contributed to the study design and supported the editing of the final manuscript. All authors approved the final version of the manuscript. 


\section{Acknowledgements}

The authors thank Angiola Desiderio for assistance with molecular diagnosis and WP primers designing, Federico Germani for help in Series I crossing experiments, Orsio Allegrucci for supporting in the maintenance of the insect colonies. We also thank Yuqing Fu, PhD, and James Mains, PhD, for their assistance with the HTA line and Sarah Peaslee for assistance in the Series $\|$ crossing experiments.

This research was supported by the Italian National Agency for New Technologies, Energy and Sustainable Economic Development (ENEA).

\section{Author details}

'Laboratory of Sustainable Management of the Agro-ecosystem, ENEA (Italian National Agency for New Technologies, Energy and Sustainable Economic Development), Via Anguillarese, 310, 00123, Rome, Italy. ${ }^{2}$ Department of Entomology, University of Kentucky, Lexington, KY 40546, USA.

Received: 11 August 2012 Accepted: 10 October 2012 Published: 12 November 2012

\section{References}

1. Benedict MQ, Levine RS, Hawley WA, Lounibos LP: Spread of the tiger: global risk of invasion by the mosquito Aedes albopictus. Vector Borne Zoonotic Dis 2007, 7:76-85.

2. Gratz NG: Critical review of the vector status of Aedes albopictus. Med Vet Entomol 2004, 18:215-227.

3. Talbalaghi A, Moutailler S, Vazeille M, Failloux A-B: Are Aedes albopictus or other mosquito species from northern Italy competent to sustain new arboviral outbreaks? Med Vet Entomol 2010, 24:83-87.

4. Sabatini A, Raineri V, Trovato G, Coluzzi M: Aedes albopictus in Italia e possibile diffusione della specie nell'area mediterranea. Parassitologia 1990, 32:301-304.

5. Romi R: Aedes albopictus in Italia: un problema sottovalutato. Ann Ist Sup Sanità 2001, 37(2):241-247.

6. Angelini R, Finarelli $A C$, Angelini $P, P O C$, Petropulacos $K$, Macini $P$, Fiorentini C, Fortuna C, Venturi G, Romi R, Majori G, Nicoletti L, Rezza G, Cassone A: An outbreak of Chikungunya fever in the province of Ravenna, Italy. Euro Surveillance 2007, 12:pii =3260.

7. La Ruche $G$, Souarès $Y$, Armengaud $A$, Peloux-Petiot $F$, Delaunay $P$, Desprès P, Lenglet A, Jourdain F, Leparc-Goffart I, Charlet F, Ollier L, Mantey K, Mollet T, Fournier JP, Torrents R, Leitmeyer K, Hilairet P, Zeller $H$, Van Bortel W, Dejour-Salamanca D, Grandadam M, Gastellu-Etchegorry M: First two autochthonous dengue virus infections in metropolitan France. Euro surveillance 2010, 15:39. Rapid communications.

8. Carrieri M, Angelini P, Venturelli C, Bellini R: Aedes albopictus (Diptera: Culicidae) population size survey in the 2007 Chikungunya outbreak area in Italy. II: estimating epidemic thresholds. J Med Entomol 2012, 49(2):388-399

9. Bellini R, Calvitti M, Medici A, Carrieri M, Celli G, Maini S: Use of the sterile insect technique against Aedes albopictus in Italy: First results of a pilot trial. In Area-wide control of insect pests: from research to field implementation. Edited by Vreysen MJB, Robinson AS, Hendrichs J, Dordrecht. The Netherlands: Springer; 2007:505-515.

10. Urbanelli S, Bellini R, Carrieri M, Sallicandro P, Celli G: Population structure of Aedes albopictus (Skuse): the mosquito which is colonizing Mediterranean countries. Heredity 2000, 84:331-337.

11. Marini F, Caputo C, Pombi M, Tarsitani G, Della Torre A: Study of Aedes albopictus dispersal in Rome, Italy, using sticky traps in mark-releaserecapture experiments. Med Vet Entomol 2010, 24(4):361-368.

12. Honòrio NA, Da Costa Silva W, Leite PJ, Goncalves JM, Lounibos LP, Lourenco-de-Oliveira R: Dispersal of Aedes aegypti and Aedes albopictus (Diptera: Culicidae) in an urban endemic dengue area in the State of Rio de Janeiro, Brazil. Mem. Inst. Oswaldo Cruz 2003, 98:191-198.

13. Bellini R, Albieri A, Balestrino F, Carrieri M, Porretta D, Urbanelli S, Calvitti M, Moretti R, Maini S: Dispersal and survival of Aedes albopictus (Diptera: Culicidae) males in Italian urban areas and significance for sterile insect technique application. J Med Entomol 2010, 47:1082-1091.

14. Alphey L, Benedict M, Bellini R, Clark GG, Dame DA, Service MW, Dobson SL: Sterile insect methods for control of mosquito-borne desease: an analysis. Vector Borne Zoonotic Dis 2010, 10:295-311.
15. Balestrino F, Medici A, Candini G, Carrieri M, Maccagnani B, Calvitti M: Maini $S$, and Bellini $R: \gamma$ ray dosimetry and mating capacity studies in the laboratory on Aedes albopictus males. J Med Entomol 2010, 47(4):581-591.

16. Floate KD, Kyei-Poku GK, Coghlin PC: Overview and relevance of Wolbachia bacteria in biocontrol research. Biocontrol Sci Technol 2006, 16:767-788.

17. Hilgenboecker K, Hammerstein P, Sclattman P: Telscowa, Werren JH: How many species are infected with Wolbachia? A statistical analysis of current data. FEMS Microbiol let 2008, 281:215-220.

18. Werren JH, Baldo L, Clark ME: Wolbachia: master manipulators of invertebrate biology. Nat Rev Microbiol 2008, 6:741-751.

19. Breeuwer JA, Werren JH: Microorganisms associated with chromosome destruction and reproductive isolation between two insect species. Nature 1993, 346:558-560.

20. Callaini G, Dallai R, Riparbelli MG: Wolbachia induced delay of paternal chromatin condensation does not prevent maternal chromosomes from entering anaphase in incompatible crosses of Drosophila simulans. J Cell Sci 1997, 110:271-280.

21. Serbus LR, Casper-Lindley C, Landmann F, Sullivan W: The Genetics and Cell Biology of Wolbachia Host Interactions. Annu Rev Genet 2008, 42:683-707.

22. Brelsfoard CL, Dobson SL: Population genetic structure of Aedes polynesiensis in the Society Islands of French Polynesia: implications for control using a Wolbachia-based autocidal strategy. Parasite Vectors 2012, 5:80.

23. Zabalou S, Apostolaki A, Livadaras I, Franz G, Robinson AS, Savakis C, Bourtzis K: Incompatible insect technique: incompatible males from a Ceratitis capitata genetic sexing strain. Entomol Exp Appl 2009, 132:232-240.

24. Brelsfoard CL, St Clair W, Dobson SL: Integration of irradiation with cytoplasmic incompatibility to facilitate a lymphatic filariasis vector elimination approach. Parasite Vectors 2009, 2:38.

25. Bourtzis K, Robinson A: Insect pest control using Wolbachia and/or irradiation. In Insect Symbiosis, Volume 2. Edited by Bourtzis K, Miller A. Boca Raton FL (USA): CRC Taylor and Francis; 2006:225-246.

26. Walker T, Johnson PH, Moreira LA, Iturbe-Ormaetxe I, Frentiu FD, McMeniman CJ: A non-virulent Wolbachia infection blocks dengue transmission and rapidly invades Aedes aegypti populations. Nature 2011, 476:454-460.

27. Hoffmann AA, Montgomery BL, Popovici J, Iturbe-Ormaetxe I, Johnson PH, Muzzi F: Successful establishment of Wolbachia in Aedes populations to suppress dengue transmission. Nature 2011, 476:450-455.

28. Rasgon JL: Mosquitoes attacked from within. Nature 2011, 476:407-408.

29. Blagrove MSC, Arias-Goeta C, Failloux AB, Sinkins SP: Wolbachia wMel induces cytoplasmic incompatibility and blocks dengue transmission in Aedes albopictus. PNAS 2012, 109:255-260.

30. Ioannidis P, Bourtzis K: Insect symbionts and applications: the paradigm of cytoplasmic incompatibility-inducing Wolbachia. Entomol Res 2007, 37:125-138.

31. Calvitti M, Moretti R, Lampazzi E, Bellini, Dobson SL: Characterization of a new Aedes albopictus (Diptera: Culicidae) -Wolbachia pipientis (Rickettsiales: Rickettsiaceae) symbiotic association generated by artificial transfer of the wPip strain from Culex pipiens (Diptera: Culicidae). $J$ Med Entomol 2010, 47:179-187.

32. Dobson SL, Rattanadechakul W: A novel technique for removing Wolbachia infections from Aedes albopictus. J Med Entomol 2001, 38:844-849.

33. Calvitti M, Moretti R, Porretta D, Bellini R, Urbanelli S: Effects on male fitness of removing Wolbachia infections from the mosquito Aedes albopictus. Med Vet Entomol 2009, 23:132-140.

34. Wiwatanaratanabutr I, Kittayapong P: Effects of crowding and temperature on Wolbachia infection density among life cycle stages of Aedes albopictus. J Invertebr Pathol 2009, 102:220-224.

35. Weeks AR, Reynolds KT, Hoffman A: Wolbachia dynamics and host effects: what has (and has not) been demonstrated. Trends Ecol Evol 2002, 176:257-262.

36. Fu Y, Gavotte L, Mercer DR, Dobson SL: Artificial triple Wolbachia infection in Aedes albopictus yields a new pattern of unidirectional cytoplasmic incompatibility. Appl Environ Microbiol 2010, 76:5887-5891.

37. Xi Z, Dean JL, Khoo C, Dobson SL: Generation of a novel Wolbachia infection in Aedes albopictus (Asian tiger mosquito) via embryonic microinjection. Insect Biochem Mol Biol 2005, 35:903-910.

38. Zhou W, Rousset R, O' Neill SL: Phylogeny and PCR-based classification of Wolbachia strains using wsp gene sequences. Proc R Soc Lond B Biol Sci 1998, 265:509-515. 
39. Braig HR, Zhou W, Dobson SL, O' Neill SL: Cloning and characterization of a gene encoding the major surface protein of the bacterial endosymbiont Wolbachia pipientis. J Bacteriol 1989, 180:2373-2378.

40. Simon C, Frati F, Beckenbach A, Crespi B, Liu H, Flook P: Evolution, weighting and phylogenetic utility of mitochondrial gene sequences and a compilation of conserved polymerase chain reaction primers. Ann Entomol Soc Am 1994, 87:651-701.

41. Poinsot D, Bourtzis K, Markakis G, Savakis C, Merçot H: Wolbachia transfer from Drosophila melanogaster to Drosophila simulans: host effect and cytoplasmic incompatibility relationships. Genetics 1998, 150:227-237.

42. Boyer S, Gilles J, Merancienne D, Lemperiere G, Fontenille D: Sexual performance of male mosquito Aedes albopictus. Med Vet Entomol 2011, 25:454-459.

43. Reynolds KT, Hoffmann AA: Male age, host effects and weak expression or non-expression of cytoplasmic incompatibility in Drosophila strains infected by maternal transmitted Wolbachia. Genet Res 2002, 80:79-87.

44. Binnington KC, Hoffman AA: Wolbachia like organisms and cytoplasmic incompatibility in Drosophila simulans. J Invertebr Pathol 1989, 54:344-352.

45. Bressac C, Rousset F: The reproductive incompatibility system in Drosophila simulans: DAPI-staining analysis of the Wolbachia symbionts in sperm cysts. J Invertebr Pathol 1993, 61:226-230.

46. Rasgon JL, Scott WT: Wolbachia and cytoplasmic incompatibility in the California Culex pipiens mosquito species complex: parameter estimates and infection dynamics in natural populations. Genetics 2003, 165:2029-2038.

47. Duron O, Fort $P$, Weill M: Influence of male aging on cytoplasmic incompatibility, sperm modification and Wolbachia density in Culex pipiens mosquitoes. Heredity 2007, 98:368-374

48. Breeuwer JA, Werren $\mathrm{JH}$ : The effect of genotypeon cytoplasmic incompatibility between two species of Nasonia. Heredity 1993, 70:428-436

49. Kittayapong P, Mongkalangoon P, Baimai V, O'Neill SL: Host age effect and expression of cytoplasmic incompatibility in field population of Wolbachia-superinfected Aedes albopictus. Heredity 2002, 88:270-274.

50. Sinkins SP, Braig HR, O'neill SL: Wolbachia superinfections and the expression of cytoplasmic incompatibility. Proc R Soc London B 1995, 261:325-330.

51. Tortosa P, Charlat S, Labbé P, Dehecq J-S, Barré H, Weill M: Wolbachia agesex-specific density in Aedes albopictus: a host evolutionary response to cytoplasmic Incompatibility? PLoS One 2010, 5:e9700.

52. Sinkins SP, Walker T, Lynd AR, Steven AR, Makepeace BL, Godfray HCJ, Parkhill J: Wolbachia variability and host effects on crossing type in Culex mosquitoes. Nature 2005, 436:257-260.

53. Zabalou S, Apostolaki A, Pattas S, Veneti Z, Paraskevopoulos C, Livadaras I, Markakis G, Brissac T, Merçot H, Bourtzis K: Multiple Rescue Factors within a Wolbachia Strain. Genetics 2008, 150:227-237.

54. Lacroix R, Delatte $H$, Hue T, Reiter P: Dispersal and survival of male and female Aedes albopictus (Diptera Culicidae) on Reunion Island. J Med Entomol 2009, 46:1117-1124.

55. Bellini R, Balestrino F, Medici A, Gentile G, Veronesi R, Carrieri M: Mating competitiveness of Aedes albopictus radio-sterilized males in large enclosures exposed to natural conditions. J Med Entomol 2012, in press.

56. Helinsky MEH, Parker AG, Knols BGJ: Radiation biology of mosquitoes. Malar J 2008, 8(2):S6. doi:10.1186/1475-2875-8-S2-S6.

57. Asman KNF, Reisen SM: Changes in the biology of Culex tarsalis (Diptera: Culicidae) associated with colonization under contrasting regimes. Environ Entomol 1987, 16:405-414.

58. Hansson B, Westerberg L: On the correlation between heterozygosity and fitness in natural populations. Mol Ecol 2002, 11:2467-2474.

59. Woodworth LM, Montgomery ME, Briscoe DA, Frankham R: Rapid genetic deterioration in captive populations: Causes and conservation implications. Conserv Genet 2002, 3:277-288.
60. Xi Z, Khoo C, Dobson SL: Wolbachia establishment and invasion in an Aedes aegypti laboratory population. Science 2005, 310:326-328.

61. Hancock PA, Sinkins SP, Godfray HC: Strategies for introducing Wolbachia to reduce transmission of mosquito-borne diseases. PLoS Negl Trop Dis 2011, 5(4):e1024

\section{doi:10.1186/1756-3305-5-254}

Cite this article as: Calvitti et al:: Wolbachia strain wPip yields a pattern of cytoplasmic incompatibility enhancing a Wolbachia-based suppression strategy against the disease vector Aedes albopictus.

Parasites \& Vectors 2012 5:254

\section{Submit your next manuscript to BioMed Central and take full advantage of:}

- Convenient online submission

- Thorough peer review

- No space constraints or color figure charges

- Immediate publication on acceptance

- Inclusion in PubMed, CAS, Scopus and Google Scholar

- Research which is freely available for redistribution

Submit your manuscript at www.biomedcentral.com/submit

C BioMed Central 